\title{
A known Iron(II) Complex in Different Nanosized Particles: Variable-Temperature Raman Study of its Spin-Crossover Behavior
}

Zoi G. Lada, ${ }^{\dagger}{ }^{\S}$ Konstantinos S. Andrikopoulos,,$" \dagger$, Athanassios Chrissanthopoulos, ${ }^{\ddagger}$ Spyros P. Perlepes, ${ }^{*} \S$ and George A. Voyiatzis ${ }^{*}{ }^{\dagger}$

$\dagger$ Institute of Chemical Engineering Sciences (ICE-HT), Foundation for Research and Technology- Hellas (FORTH), , Platani, P.O. Box 1414, 26504 Patras, Greece

Laboratory of Inorganic Chemistry, Department of Chemistry, National and Kapodistrian University of Athens, Panepistimiopolis, 15771 Zografou, Athens, Greece

$\S$ Department of Chemistry, University of Patras, 26504 Patras, Greece 
Table S1. Atoms' cartesian coordinates at equilibrium geometry: molecule: abpt (crystalline state conformation), theory/basis set: B3LYP/TZVp.

$\begin{array}{llrr}\mathrm{H} & -0.84900000 & -1.96400000 & -0.01900000 \\ \mathrm{~N} & -0.00400000 & -1.58500000 & -0.45300000 \\ \mathrm{H} & 0.85000000 & -1.96400000 & -0.03700000 \\ \mathrm{~N} & -0.00100000 & -0.20900000 & -0.13900000 \\ \mathrm{C} & 1.09000000 & 0.61600000 & -0.06800000 \\ \mathrm{~N} & 0.67800000 & 1.87800000 & 0.04400000 \\ \mathrm{~N} & -0.67800000 & 1.87900000 & 0.04200000 \\ \mathrm{C} & -1.09100000 & 0.61700000 & -0.07000000 \\ \mathrm{C} & 2.49400000 & 0.19000000 & -0.05800000 \\ \mathrm{~N} & 2.74400000 & -1.12500000 & 0.09600000 \\ \mathrm{C} & 4.02300000 & -1.52200000 & 0.13400000 \\ \mathrm{H} & 4.18600000 & -2.59000000 & 0.25900000 \\ \mathrm{C} & 3.51400000 & 1.14700000 & -0.17600000 \\ \mathrm{H} & 3.25700000 & 2.19300000 & -0.29500000 \\ \mathrm{C} & 4.83600000 & 0.71700000 & -0.13100000 \\ \mathrm{H} & 5.64900000 & 1.43400000 & -0.21800000 \\ \mathrm{C} & 5.10200000 & -0.64500000 & 0.02700000 \\ \mathrm{H} & 6.11900000 & -1.02400000 & 0.06700000 \\ \mathrm{C} & -2.49400000 & 0.19100000 & -0.05800000 \\ \mathrm{~N} & -2.74400000 & -1.12300000 & 0.10300000 \\ \mathrm{C} & -4.02200000 & -1.52000000 & 0.14400000 \\ \mathrm{H} & -4.18400000 & -2.58800000 & 0.27700000 \\ \mathrm{C} & -3.51500000 & 1.14700000 & -0.18000000 \\ \mathrm{H} & -3.25900000 & 2.19300000 & -0.30400000 \\ \mathrm{C} & -4.83600000 & 0.71600000 & -0.13500000 \\ \mathrm{H} & -5.64900000 & 1.43100000 & -0.23000000 \\ \mathrm{C} & -5.10200000 & -0.64600000 & 0.03000000 \\ \mathrm{H} & -6.11900000 & -1.02400000 & 0.06700000\end{array}$

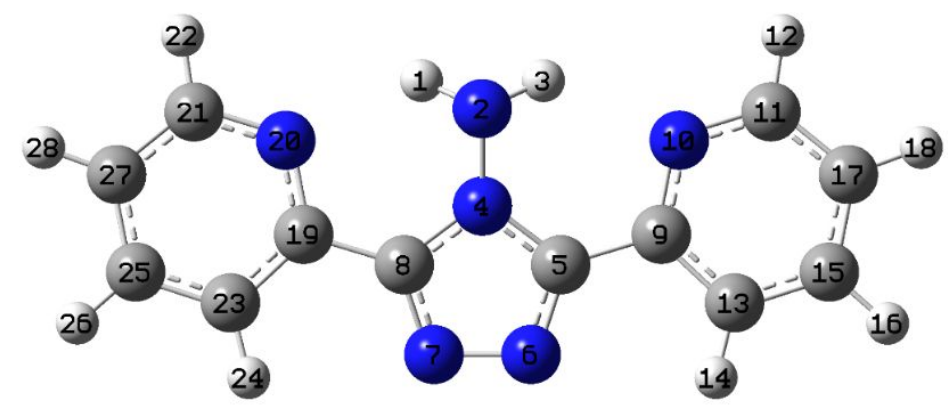

Figure S1. Drawing of the optimized structure of the abpt molecular unit (atoms' numbering as in Table S1). The molecule is in the conformation that appears in its crystal structure (ref. 24a of the main ms.).

The order of the atoms in the left column of Table S1 corresponds exactly to the numbering scheme of the atoms in the optimized structure (Fig. S1). For example the $\mathrm{N}$ atom in the 4th line is $\mathrm{N} 4$. 
Table S2. Atoms' cartesian coordinates at equilibrium geometry: molecule: abpt (ligand conformation), theory/basis set: B3LYP/TZVp.

$\begin{array}{lrlr}\mathrm{H} & 0.2363369954 & 0.0474911272 & 2.9107250011 \\ \mathrm{~N} & 0.9866045721 & -0.1911562131 & 2.2599162119 \\ \mathrm{H} & 1.4555886744 & 0.6743810527 & 2.0018033681 \\ \mathrm{~N} & 0.3321027521 & -0.6815281538 & 1.1088590251 \\ \mathrm{C} & 0.9196874387 & -1.3829894359 & 0.0889144715 \\ \mathrm{~N} & 0.0215730421 & -1.6335459731 & -0.8447168405 \\ \mathrm{~N} & -1.1651031971 & -1.1109320214 & -0.4347444341 \\ \mathrm{C} & -0.9796184119 & -0.5269933759 & 0.7326121493 \\ \mathrm{C} & 2.3301172950 & -1.8106554448 & 0.0446891146 \\ \mathrm{~N} & 2.9252488593 & -1.7455239688 & -1.1531626677 \\ \mathrm{C} & 4.1785112477 & -2.1825028058 & -1.2514228008 \\ \mathrm{H} & 4.6269769276 & -2.1152386203 & -2.2375011984 \\ \mathrm{C} & 2.9760495355 & -2.3034211138 & 1.1832753240 \\ \mathrm{H} & 2.4617762746 & -2.3247967259 & 2.1330492202 \\ \mathrm{C} & 4.2839445610 & -2.7508601519 & 1.0627151018 \\ \mathrm{H} & 4.8079716971 & -3.1433831420 & 1.9255955886 \\ \mathrm{C} & 4.9031098587 & -2.6946021612 & -0.1794088601 \\ \mathrm{H} & 5.9211158072 & -3.0350943658 & -0.3190067231 \\ \mathrm{C} & -2.0321591998 & 0.1302942572 & 1.5062637295 \\ \mathrm{~N} & -1.7601093396 & 0.4819907597 & 2.7736611855 \\ \mathrm{C} & -2.7243486023 & 1.0645643795 & 3.4898404030 \\ \mathrm{H} & -2.4643005584 & 1.3323934733 & 4.5082398567 \\ \mathrm{C} & -3.2840665321 & 0.3522263586 & 0.9187771483 \\ \mathrm{H} & -3.4483861204 & 0.0440961096 & -0.1041022034 \\ \mathrm{C} & -4.2729496614 & 0.9531079916 & 1.6775584888 \\ \mathrm{H} & -5.2515605912 & 1.1353923082 & 1.2509177082 \\ \mathrm{C} & -3.9937429194 & 1.3212157063 & 2.9917718855 \\ \mathrm{H} & -4.7401707246 & 1.7939242594 & 3.6162651566\end{array}$

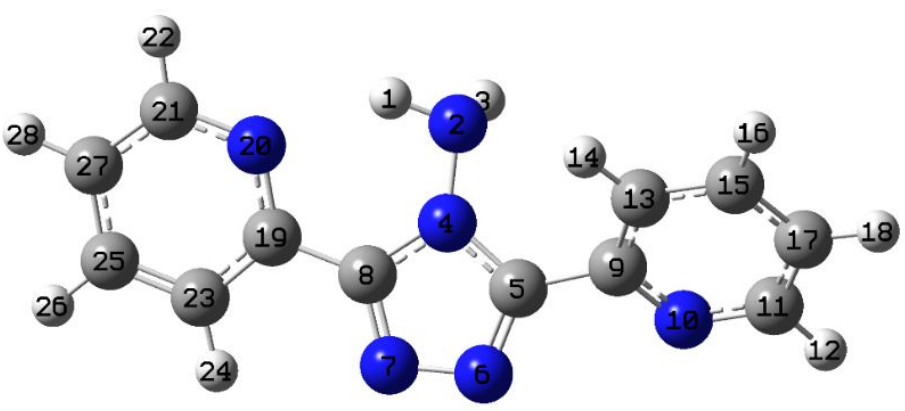

Figure S2. Drawing of the optimized molecular structure of the ligand abpt (atoms' numbering as in Table S2). The molecule is in the conformation that appears in the crystal structure of complex 1.

The order of the atoms in the left column of Table S2 corresponds exactly to the numbering scheme of the atoms in the optimized structure (Fig. S2). For example the $\mathrm{C}$ atom in the 8 th line is $\mathrm{C} 8$. 
Table S3. Atoms' cartesian coordinates at equilibrium geometry: molecule: LS $\left[\mathrm{Fe}\left\{\mathrm{N}(\mathrm{CN})_{2}\right\}_{2}(\text { abpt })_{2}\right]$, theory/basis set: $\mathrm{B} 3 \mathrm{LYP} / \mathrm{TZVp}$.

\begin{tabular}{|c|c|}
\hline $\mathrm{Fe}$ & 1.9677870000 \\
\hline $\mathrm{N}$ & 1.7247323045 \\
\hline $\mathrm{N}$ & 0.9659131647 \\
\hline $\mathrm{N}$ & 2.5355549655 \\
\hline $\mathrm{N}$ & 3.8370695997 \\
\hline $\mathrm{C}$ & 2.8084749872 \\
\hline $\mathrm{C}$ & 0.4498212458 \\
\hline $\mathrm{N}$ & 2.5250836039 \\
\hline $\mathrm{C}$ & 1.2179148357 \\
\hline $\mathrm{N}$ & 0.7301466908 \\
\hline $\mathrm{N}$ & 3.4644426131 \\
\hline $\mathrm{H}$ & 3.864024926 \\
\hline $\mathrm{H}$ & 2.9120603062 \\
\hline $\mathrm{C}$ & 3.555064678 \\
\hline $\mathrm{C}$ & -0.778926438 \\
\hline $\mathrm{H}$ & -1.147601402 \\
\hline $\mathrm{C}$ & 4.011769952 \\
\hline $\mathrm{C}$ & -0.967777035 \\
\hline $\mathrm{H}$ & -1.496873824 \\
\hline $\mathrm{N}$ & 3.2676274872 \\
\hline $\mathrm{C}$ & -1.4954684940 \\
\hline $\mathrm{H}$ & -2.452242882 \\
\hline $\mathrm{C}$ & 2.883533705 \\
\hline $\mathrm{C}$ & 6.263020239 \\
\hline $\mathrm{H}$ & 7.204337628 \\
\hline $\mathrm{C}$ & 5.205474445 \\
\hline $\mathrm{H}$ & 5.283112519 \\
\hline $\mathrm{C}$ & 4.862788037 \\
\hline $\mathrm{H}$ & 4.682144835 \\
\hline $\mathrm{C}$ & 0.265722910 \\
\hline $\mathrm{H}$ & 0.712140882 \\
\hline $\mathrm{C}$ & 6.091467759 \\
\hline $\mathrm{H}$ & 6.891595760 \\
\hline $\mathrm{N}$ & 3.827604543 \\
\hline $\mathrm{N}$ & 2.210841695 \\
\hline $\mathrm{N}$ & 2.969660835 \\
\hline $\mathrm{N}$ & 1.40001903 \\
\hline $\mathrm{N}$ & 0.098504400 \\
\hline $\mathrm{C}$ & 1.127099012 \\
\hline $\mathrm{C}$ & 3.485752754 \\
\hline $\mathrm{N}$ & 1.41049039 \\
\hline $\mathrm{C}$ & 2.717659164 \\
\hline $\mathrm{N}$ & 3.205427309 \\
\hline $\mathrm{N}$ & 0.47113138 \\
\hline $\mathrm{H}$ & 0.071549073 \\
\hline $\mathrm{H}$ & 1.0235136 \\
\hline $\mathrm{C}$ & \\
\hline
\end{tabular}

$\begin{array}{rr}4.3828201029 & 0.0000000000 \\ 3.7053772726 & 1.8736408129 \\ 2.4311926496 & 6.1308606466 \\ 3.2690488850 & 3.8448147651 \\ 4.7267838577 & 0.7606321899 \\ 3.7999807679 & 2.6223219107 \\ 2.3031712903 & 4.8985878263 \\ 2.5792761736 & -0.5711765699 \\ 2.8726517548 & 3.7894924239 \\ 3.1448016532 & 2.5958632383 \\ 3.1331573342 & 4.8939114731 \\ 2.1987277690 & 4.8301156523 \\ 3.1659733339 & 5.7510722274 \\ -0.0562296565 & -2.4683441580 \\ 1.6840293697 & 4.6548833363 \\ 1.6018646724 & 3.6425388469 \\ 4.3859727729 & 2.0605629553 \\ 1.3286800971 & 7.0150626167 \\ 0.9605566771 & 7.8838563628 \\ 0.3524417887 & -1.2533076372 \\ 1.1945443473 & 5.7351793170 \\ 0.7117422275 & 5.5811640101 \\ 1.5343505200 & -0.9561760924 \\ 5.1794143264 & 2.0492321291 \\ 5.3638161203 & 2.5508401288 \\ 4.6024203787 & 2.7400185579 \\ 4.3317857345 & 3.7821284219 \\ 5.2751884419 & 0.1078232295 \\ 5.5276574255 & -0.9277186506 \\ 1.9514833539 & 7.1593240312 \\ 2.0766796336 & 8.1394103058 \\ 5.5138649409 & 0.7146718994 \\ 5.9595719375 & 0.1394234258 \\ -0.4984936776 & -3.5100782994 \\ 5.0602629333 & -1.8736408129 \\ 6.3344475563 & -6.1308606466 \\ 5.4965913209 & -3.8448147651 \\ 4.0388563482 & -0.7606321899 \\ 4.9656594380 & -2.6223219107 \\ 6.4624689155 & -4.8985878263 \\ 6.1863640323 & 0.5711765699 \\ 5.8929884511 & -3.7894924239 \\ 5.6208385526 & -2.5958632383 \\ 5.6324828717 & -4.8939114731 \\ 6.5669124369 & -4.8301156523 \\ 5.5996668720 & -5.7510722274 \\ 8.8218698624 & 2.4683441580\end{array}$




$\begin{array}{lrlr}\mathrm{C} & 4.7145004386 & 7.0816108362 & -4.6548833363 \\ \mathrm{H} & 5.0831754029 & 7.1637755335 & -3.6425388469 \\ \mathrm{C} & -0.0761959523 & 4.3796674329 & -2.0605629553 \\ \mathrm{C} & 4.9033510353 & 7.4369601088 & -7.0150626167 \\ \mathrm{H} & 5.4324478246 & 7.8050835287 & -7.8838563628 \\ \mathrm{~N} & 0.6679465128 & 8.4131984172 & 1.2533076372 \\ \mathrm{C} & 5.4310424940 & 7.5710958586 & -5.7351793170 \\ \mathrm{H} & 6.3878168821 & 8.0538979783 & -5.5811640101 \\ \mathrm{C} & 1.0520402945 & 7.2312896859 & 0.9561760924 \\ \mathrm{C} & -2.3274462395 & 3.5862258795 & -2.0492321291 \\ \mathrm{H} & -3.2687636282 & 3.4018240856 & -2.5508401288 \\ \mathrm{C} & -1.2699004454 & 4.1632198272 & -2.7400185579 \\ \mathrm{H} & -1.3475385194 & 4.4338544714 & -3.7821284219 \\ \mathrm{C} & -0.9272140377 & 3.4904517639 & -0.1078232295 \\ \mathrm{H} & -0.7465708359 & 3.2379827804 & 0.9277186506 \\ \mathrm{C} & 3.6698510894 & 6.8141568520 & -7.1593240312 \\ \mathrm{H} & 3.2234331175 & 6.6889605723 & -8.1394103058 \\ \mathrm{C} & -2.1558937597 & 3.2517752650 & -0.7146718994 \\ \mathrm{H} & -2.9560217601 & 2.8060682684 & -0.1394234258 \\ \mathrm{~N} & 0.1079694563 & 9.2641338835 & 3.5100782994\end{array}$

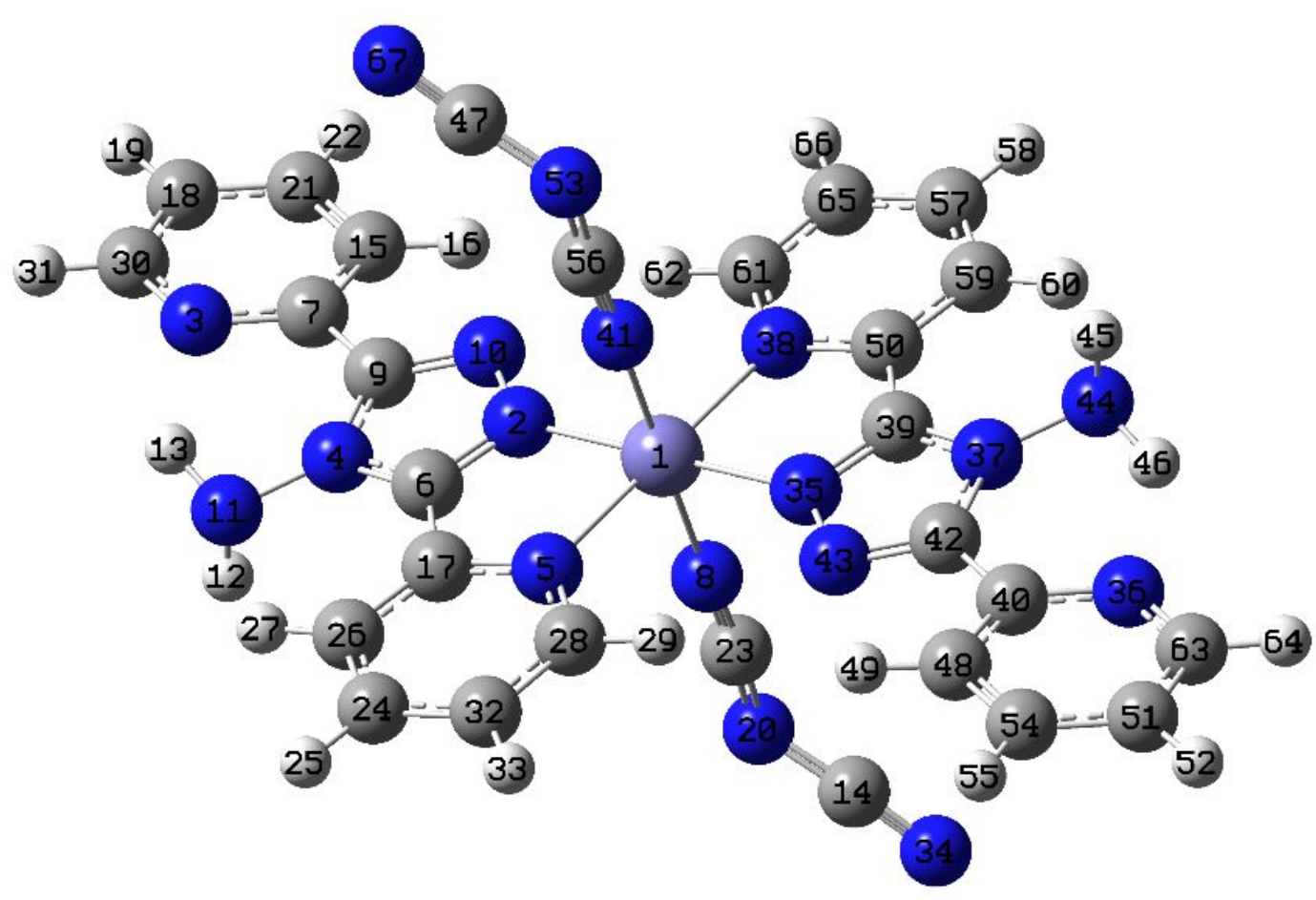

Figure S3. Drawing of the optimized molecular structure of the LS Fe(II) complex $\left[\mathrm{Fe}\left\{\mathrm{N}(\mathrm{CN})_{2}\right\}_{2}(\mathrm{abpt})_{2}\right]$ having $C_{\mathrm{i}}$ symmetry (atoms' numbering as in Table $\mathbf{S 3}$ ).

The order of the atoms in the left column of Table S3 corresponds exactly to the numbering scheme of the atoms in the optimized structure (Fig. S3). For example the $\mathrm{N}$ atom in the 12th line is $\mathrm{H} 12$. 


\section{Analysis of the Calculated Vibrational Modes}

Assignments of the vibrational modes have been performed by means of potential energy distribution (PED) analysis using the Vibrational Energy Distribution Analysis (VEDA) software [S1]. The calculated normal modes are represented by internal coordinates giving a more intuitive view of the atoms' movements during vibration. Each of the internal coordinates is expressed as a superposition of several local modes (see last column of Table S4) of two, three and four atoms connected by bonds for stretching (STRE), bending (BEND), and torsional (TORS) or out-of-plane (OUT) local modes, respectively.

Table S4. Calculated harmonic wavenumbers $\left(\mathrm{cm}^{-1}\right)$ at B3LYP level of theory using the TZVp basis sets for the LS $\mathrm{Fe}(\mathrm{II})$ complex $\left[\mathrm{Fe}\left\{\mathrm{N}(\mathrm{CN})_{2}\right\}_{2}(\mathrm{abpt})_{2}\right]$ at $C_{\mathrm{i}}$ symmetry. Calculated IR intensities are in $\mathrm{km} \cdot \mathrm{mol}^{-1}$ and Raman activities are in $\AA^{4} \cdot(\mathrm{amu})^{-1}$. Calculations have been performed at the optimized geometry at the B3LYP/TZVp level of theory. PED assignment of the vibrational modes using internal coordinates are shown inTable S5.

\begin{tabular}{|c|c|c|c|c|c|}
\hline no & Sym & $\begin{array}{r}\text { Wavenumber } \\
{\left[\mathrm{cm}^{-1}\right]}\end{array}$ & $\begin{array}{r}\text { IR } \\
\text { Intensity }\end{array}$ & $\begin{array}{l}\text { Raman } \\
\text { activity }\end{array}$ & Assignment [scaled frequencies and PED local modes] \\
\hline 1 & $A_{g}$ & 3.87 & 0.00 & 20.28 & $4.46 \quad s 15427 \quad$ s155 $-15 \quad$ s156 $-17 \quad$ s157 10 \\
\hline 2 & $\overline{A_{u}}$ & 5.21 & 10.70 & 0.00 & $5.38 \quad s 15419$ s155 $31 \quad s 156-10 \quad s 157-18$ \\
\hline 3 & $A_{u}$ & 9.16 & 2.76 & 0.00 & $9.16 \quad$ s128 $-18 \quad s 19414$ s195 21 \\
\hline 4 & $A_{g}$ & 10.36 & 0.00 & 10.40 & 10.28 s96 -39 s129 11 s185 10 \\
\hline 5 & $A_{u}$ & 14.61 & 11.46 & 0.00 & $14.62 \quad s 9565 \quad s 12911$ \\
\hline 6 & $A_{u}$ & 22.42 & 2.89 & 0.00 & $22.42 \quad s 161-28$ s181-21 \\
\hline 7 & $A_{g}$ & 25.68 & 0.00 & 6.25 & 25.40 s130-11 s156-14 s157 12 \\
\hline 8 & $A_{u}$ & 26.78 & 0.32 & 0.00 & $27.70 \quad$ s95 $14 \quad$ s155 $-111 \quad$ s156 $20 \quad$ s157 $23 \quad$ s184 14 \\
\hline 9 & $A_{g}$ & 32.55 & 0.00 & 3.67 & 32.52 s171-18 s174 18 \\
\hline 10 & $A_{u}$ & 42.62 & 10.61 & 0.00 & 42.63 s171-11 s174-12 s187 $12 \quad$ s188 -16 \\
\hline 11 & $A_{u}$ & 43.46 & 0.10 & 0.00 & 44.59 s96 17 s163 -11 s165 $12 \quad$ s186 15 s187 14 \\
\hline 12 & $A_{g}$ & 44.73 & 0.00 & 6.09 & $44.94 \quad s 7230 \quad s 13115$ \\
\hline 13 & $A_{u}$ & 52.37 & 1.17 & 0.00 & $52.39 \quad s 163-19 \quad s 165-19 \quad$ s186 $-10 \quad$ s187 15 \\
\hline 14 & $A_{g}$ & 65.66 & 0.00 & 4.62 & 65.77 s65 -16 \\
\hline 15 & $A_{g}$ & 73.09 & 0.00 & 3.88 & $73.11 \quad s 6513$ \\
\hline 16 & $A_{g}$ & 87.18 & 0.00 & 4.03 & 86.71 s177 -37 \\
\hline 17 & $A_{u}$ & 94.97 & 3.35 & 0.00 & 95.01 s99 -10 s100 10 \\
\hline 18 & $A_{g}$ & 103.96 & 0.00 & 5.58 & 102.95 \\
\hline 19 & $A_{u}$ & 108.41 & 0.56 & 0.00 & 108.41 \\
\hline 20 & $A_{u}$ & 119.53 & 9.49 & 0.00 & 119.67 s72 16 \\
\hline 21 & $A_{g}$ & 127.68 & 0.00 & 9.50 & 125.16 \\
\hline 22 & $A_{u}$ & 132.86 & 6.87 & 0.00 & $132.88 \quad s 72-10 \quad s 17814$ \\
\hline 23 & $A_{g}$ & 137.35 & 0.00 & 3.20 & $138.95 \quad s 6537$ \\
\hline
\end{tabular}




\begin{tabular}{|c|c|c|c|c|c|}
\hline 24 & $A_{g}$ & 147.05 & 0.00 & 9.14 & 168.12 \\
\hline 25 & $A_{g}$ & 168.34 & 0.00 & 5.36 & 180.68 s33 $37 \quad$ s184 12 \\
\hline 26 & $A_{u}$ & 185.03 & 1.80 & 0.00 & 202.94 s64 24 s104 13 \\
\hline 27 & $A_{u}$ & 204.82 & 1.02 & 0.00 & 207.15 s62 -19 s128 -11 \\
\hline 28 & $A_{u}$ & 208.18 & 3.01 & 0.00 & 216.48 s63 -13 \\
\hline 29 & $A_{g}$ & 221.18 & 0.00 & 6.83 & 218.38 \\
\hline 30 & $A_{g}$ & 225.27 & 0.00 & 1.69 & 230.81 \\
\hline 31 & $A_{u}$ & 230.82 & 2.19 & 0.00 & $233.21 \quad s 6335$ \\
\hline 32 & $A_{g}$ & 235.42 & 0.00 & 10.45 & 242.00 \\
\hline 33 & $A_{u}$ & 242.35 & 2.26 & 0.00 & 250.05 s63 -22 \\
\hline 34 & $A_{g}$ & 252.22 & 0.00 & 17.59 & 266.41 s133 40 \\
\hline 35 & $A_{u}$ & 267.69 & 26.98 & 0.00 & $267.78 \quad s 13547$ \\
\hline 36 & $A_{g}$ & 268.24 & 0.00 & 5.74 & 274.32 s133 -22 \\
\hline 37 & $A_{u}$ & 275.60 & 11.07 & 0.00 & $283.01 \quad s 58-11 \quad s 133-12 \quad s 13511$ \\
\hline 38 & $A_{g}$ & 283.04 & 0.00 & 3.30 & 286.34 s33 -17 s184 16 \\
\hline 39 & $A_{u}$ & 289.41 & 31.16 & 0.00 & $289.72 \quad s 182-16 \quad s 183-12$ \\
\hline 40 & $A_{g}$ & 294.71 & 0.00 & 13.78 & $294.52 \quad s 182 \quad 17 \quad s 183-18$ \\
\hline 41 & $A_{u}$ & 317.77 & 12.24 & 0.00 & 319.85 s126 28 s193 -10 \\
\hline 42 & $A_{g}$ & 322.01 & 0.00 & 10.51 & 322.17 s127 26 \\
\hline 43 & $A_{u}$ & 334.29 & 6.29 & 0.00 & $334.48 \quad s 127-10$ \\
\hline 44 & $A_{u}$ & 334.60 & 4.31 & 0.00 & 341.72 s126-11 s127-13 \\
\hline 45 & $A_{g}$ & 342.00 & 0.00 & 8.79 & 374.07 s101 19 \\
\hline 46 & $A_{u}$ & 384.88 & 4.80 & 0.00 & $385.16 \quad s 6226 \quad s 64-10 \quad s 128-11$ \\
\hline 47 & $A_{u}$ & 389.46 & 1.89 & 0.00 & $389.82 \quad s 62-10 \quad s 64-16$ \\
\hline 48 & $A_{g}$ & 399.23 & 0.00 & 1.73 & 404.07 s108 12 s111 23 \\
\hline 49 & $A_{u}$ & 412.42 & 2.30 & 0.00 & $413.02 \quad$ s172 $17 \quad$ s175 -19 \\
\hline 50 & $A_{g}$ & 416.36 & 0.00 & 3.06 & 416.49 s172 -45 s175 -36 \\
\hline 51 & $A_{u}$ & 416.59 & 10.17 & 0.00 & 428.26 s16611 s169 13 \\
\hline 52 & $A_{g}$ & 443.56 & 0.00 & 0.87 & 451.46 s55 13 s101 18 \\
\hline 53 & $A_{u}$ & 459.83 & 11.94 & 0.00 & $460.38 \quad s 166-18 \quad s 16919 \quad$ s188 -17 \\
\hline 54 & $A_{g}$ & 462.68 & 0.00 & 25.07 & 463.75 s54 -24 \\
\hline 55 & $A_{u}$ & 465.47 & 3.38 & 0.00 & 471.58 \\
\hline 56 & $A_{g}$ & 488.17 & 0.00 & 1.28 & 489.71 s164 -11 \\
\hline 57 & $A_{u}$ & 493.62 & 1.33 & 0.00 & $493.62 \quad s 164-15 \quad s 167-12 \quad s 170-11$ \\
\hline 58 & $A_{g}$ & 508.43 & 0.00 & 5.06 & 513.21 s167-16 s170 -15 \\
\hline 59 & $A_{u}$ & 513.20 & 1.81 & 0.00 & 519.00 s93 12 s97 -10 \\
\hline 60 & $A_{g}$ & 521.39 & 0.00 & 8.10 & $522.29 \quad s 94-41 \quad s 9828$ \\
\hline 61 & $A_{u}$ & 522.46 & 17.92 & 0.00 & $522.81 \quad s 9330 \quad$ s97 -20 \\
\hline 62 & $A_{g}$ & 527.90 & 0.00 & 2.57 & 527.89 s152 78 s153 12 \\
\hline 63 & $A_{u}$ & 527.90 & 31.95 & 0.00 & $528.03 \quad s 152-10$ s153 75 \\
\hline 64 & $A_{u}$ & 544.54 & 7.94 & 0.00 & $544.55 \quad s 15835 \quad s 15940$ \\
\hline 65 & $A_{g}$ & 544.73 & 0.00 & 9.14 & $544.72 \quad s 15843$ s159-35 \\
\hline 66 & $A_{g}$ & 629.27 & 0.00 & 7.54 & 561.57 s192 -10 \\
\hline
\end{tabular}




\begin{tabular}{|c|c|c|c|c|c|}
\hline 67 & $A_{u}$ & 634.27 & 24.25 & 0.00 & $632.54 \quad s 6812 \quad s 102-13 \quad s 12610 \quad s 131-11$ \\
\hline 68 & $A_{g}$ & 652.47 & 0.00 & 7.79 & $641.24 \quad s 70-23 \quad s 11323$ \\
\hline 69 & $A_{g}$ & 655.60 & 0.00 & 34.01 & 654.32 s102-10 s106-14 s109 20 s126-10 \\
\hline 70 & $A_{u}$ & 655.89 & 29.81 & 0.00 & $655.66 \quad s 93 \quad 15 \quad s 9412 \quad s 9721 \quad s 9818 \quad s 9913 \quad s 10011$ \\
\hline 71 & $A_{u}$ & 656.00 & 25.48 & 0.00 & $655.98 \quad s 93-14 \quad s 9414 \quad s 97-20 \quad s 9821 \quad s 99-12 \quad s 10012$ \\
\hline 72 & $A_{u}$ & 658.36 & 5.89 & 0.00 & 657.98 s116 19 \\
\hline 73 & $A_{g}$ & 660.57 & 0.00 & 47.08 & $660.20 \quad s 10521 \quad s 10610 \quad s 107-32 \quad s 11714$ \\
\hline 74 & $A_{g}$ & 684.54 & 0.00 & 5.13 & 679.55 s61 $-12 \quad s 12513$ \\
\hline 75 & $A_{u}$ & 688.26 & 11.86 & 0.00 & 686.82 s160 49 \\
\hline 76 & $A_{g}$ & 690.39 & 0.00 & 0.78 & 691.38 s162-29 \\
\hline 77 & $A_{u}$ & 694.63 & 30.07 & 0.00 & $692.43 \quad s 179-13 \quad s 18031$ \\
\hline 78 & $A_{g}$ & 699.62 & 0.00 & 19.45 & 694.74 s179-36 s180 -30 \\
\hline 79 & $A_{u}$ & 703.16 & 2.19 & 0.00 & 699.70 \\
\hline 80 & $A_{g}$ & 729.67 & 0.00 & 1.26 & $721.39 \quad$ s60 17 \\
\hline 81 & $A_{u}$ & 731.88 & 2.69 & 0.00 & $729.83 \quad s 167-10 \quad s 18915$ s19132 \\
\hline 82 & $A_{u}$ & 746.81 & 3.81 & 0.00 & 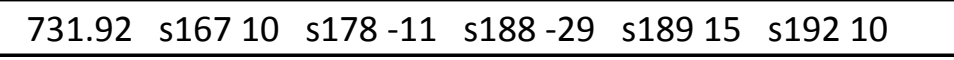 \\
\hline 83 & $A_{g}$ & 748.37 & 0.00 & 55.53 & $746.95 \quad s 6112$ \\
\hline 84 & $A_{u}$ & 752.30 & 12.32 & 0.00 & 752.16 s173 52 \\
\hline 85 & $A_{g}$ & 753.69 & 0.00 & 40.32 & $752.97 \quad s 16813$ s173 14 s176 -34 \\
\hline 86 & $A_{g}$ & 768.42 & 0.00 & 5.31 & $764.70 \quad s 6010 \quad s 11021$ \\
\hline 87 & $A_{u}$ & 768.93 & 36.10 & 0.00 & $768.48 \quad$ s55 $12 \quad$ s114 19 \\
\hline 88 & $A_{g}$ & 783.07 & 0.00 & 1.42 & $783.83 \quad s 143-42 \quad s 14730$ \\
\hline 89 & $A_{u}$ & 783.83 & 52.72 & 0.00 & 790.55 s136 25 s145 -21 \\
\hline 90 & $A_{g}$ & 791.01 & 0.00 & 3.07 & 791.28 s136-20 s145-28 s176-11 \\
\hline 91 & $A_{u}$ & 791.40 & 117.95 & 0.00 & $846.94 \quad s 6930$ \\
\hline 92 & $A_{g}$ & 916.31 & 0.00 & 2.83 & 910.63 s142 -36 s146 -29 \\
\hline 93 & $A_{u}$ & 916.33 & 0.48 & 0.00 & 916.34 s142 -42 s146 41 \\
\hline 94 & $A_{g}$ & 925.44 & 0.00 & 0.33 & 924.98 s140 34 s148 -39 \\
\hline 95 & $A_{u}$ & 925.49 & 1.57 & 0.00 & 925.50 s140 34 s148 41 \\
\hline 96 & $A_{u}$ & 938.13 & 32.28 & 0.00 & $938.14 \quad s 27-38 \quad s 2838$ \\
\hline 97 & $A_{g}$ & 938.79 & 0.00 & 39.85 & 938.81 s27 38 s28 38 \\
\hline 98 & $A_{g}$ & 984.30 & 0.00 & 1.00 & 981.73 s137 33 s150 -37 \\
\hline 99 & $A_{u}$ & 984.31 & 0.93 & 0.00 & $984.31 \quad$ s137 $37 \quad$ s150 37 \\
\hline 100 & $A_{g}$ & 1000.30 & 0.00 & 1.06 & 1000.30 s14140 s14932 s151 13 \\
\hline 101 & $A_{u}$ & 1001.07 & 1.89 & 0.00 & 1001.08 s141-38 $\quad$ s149 35 s151 10 \\
\hline 102 & $A_{g}$ & 1006.40 & 0.00 & 33.46 & 1006.28 s53 $-12 \quad s 6732$ \\
\hline 103 & $A_{u}$ & 1008.15 & 1.49 & 0.00 & 1008.29 s139-37 s151-27 \\
\hline 104 & $A_{g}$ & 1008.38 & 0.00 & 0.83 & $1009.24 \quad s 139-38 \quad s 15131$ \\
\hline 105 & $A_{u}$ & 1009.24 & 0.14 & 0.00 & 1013.36 s144 -53 \\
\hline 106 & $A_{g}$ & 1013.55 & 0.00 & 0.86 & 1013.44 s137 19 s138 63 \\
\hline 107 & $A_{u}$ & 1013.58 & 0.14 & 0.00 & 1013.97 s122 12 s144 16 \\
\hline 108 & $A_{u}$ & 1017.76 & 5.17 & 0.00 & 1017.75 s48 $16 \quad$ s70 19 s113 11 \\
\hline 109 & $A_{g}$ & 1017.78 & 0.00 & 599.48 & 1018.79 s68 14 \\
\hline
\end{tabular}




\begin{tabular}{|c|c|c|c|c|c|}
\hline 110 & $A_{u}$ & 1031.46 & 4.92 & 0.00 & 1033.59 s49 $-14 \quad s 6911$ \\
\hline 111 & $A_{g}$ & 1033.73 & 0.00 & 407.53 & 1047.39 s107 10 s134 -18 \\
\hline 112 & $A_{g}$ & 1056.55 & 0.00 & 31.75 & 1055.79 s75 16 s132 38 \\
\hline 113 & $A_{u}$ & 1057.16 & 264.88 & 0.00 & $1061.30 \quad$ s48 $14 \quad$ s112 13 \\
\hline 114 & $A_{u}$ & 1065.82 & 5.82 & 0.00 & 1066.20 s44 50 \\
\hline 115 & $A_{g}$ & 1066.39 & 0.00 & 90.60 & $1069.66 \quad s 4826 \quad s 8710$ \\
\hline 116 & $A_{u}$ & 1077.54 & 21.35 & 0.00 & 1080.08 s49 $18 \quad s 7115$ \\
\hline 117 & $A_{g}$ & 1080.25 & 0.00 & 49.84 & 1085.81 s45 -13 s49 10 \\
\hline 118 & $A_{g}$ & 1093.42 & 0.00 & 316.70 & $1094.31 \quad s 45-10 \quad s 84-17$ \\
\hline 119 & $A_{u}$ & 1094.42 & 0.67 & 0.00 & $1105.73 \quad s 4518$ s56 $11 \quad$ s84 14 \\
\hline 120 & $A_{u}$ & 1122.60 & 16.30 & 0.00 & 1123.51 s47-22 s86 31 \\
\hline 121 & $A_{g}$ & 1123.61 & 0.00 & 12.08 & $1124.26 \quad s 43-25 \quad s 7938$ \\
\hline 122 & $A_{g}$ & 1135.24 & 0.00 & 139.59 & 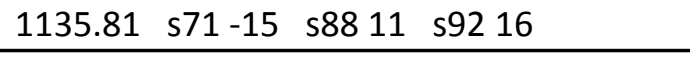 \\
\hline 123 & $A_{u}$ & 1136.69 & 1.43 & 0.00 & 1154.72 s57 -13 s103 10 \\
\hline 124 & $A_{g}$ & 1181.54 & 0.00 & 413.00 & $1181.70 \quad$ s38 $-11 \quad$ s57 $36 \quad$ s81 17 \\
\hline 125 & $A_{u}$ & 1183.28 & 1.23 & 0.00 & $1183.89 \quad s 3918 \quad s 78-15 \quad s 8551$ \\
\hline 126 & $A_{g}$ & 1183.92 & 0.00 & 81.67 & $1183.93 \quad$ s34 $17 \quad$ s78 $51 \quad$ s85 14 \\
\hline 127 & $A_{u}$ & 1183.93 & 10.92 & 0.00 & 1184.89 s56 $24 \quad s 8818$ \\
\hline 128 & $A_{g}$ & 1190.95 & 0.00 & 108.87 & $1191.35 \quad s 5615 \quad s 88-14 \quad s 9215$ \\
\hline 129 & $A_{u}$ & 1191.42 & 14.05 & 0.00 & 1192.25 s57 $-10 \quad s 8147$ \\
\hline 130 & $A_{g}$ & 1203.32 & 0.00 & 27.77 & 1203.56 \\
\hline 131 & $A_{u}$ & 1203.66 & 6.07 & 0.00 & 1211.42 s53 15 \\
\hline 132 & $A_{g}$ & 1290.11 & 0.00 & 103.18 & 1290.90 s30-14 s31 13 s40-11 \\
\hline 133 & $A_{u}$ & 1291.03 & 15.14 & 0.00 & 1294.12 s29 44 s3111 s34 -16 \\
\hline 134 & $A_{g}$ & 1295.17 & 0.00 & 15.35 & 1295.26 s29 12 s31-33 s39 15 \\
\hline 135 & $A_{u}$ & 1295.26 & 31.49 & 0.00 & 1308.81 s35 25 \\
\hline 136 & $A_{u}$ & 1311.48 & 3.94 & 0.00 & 1311.39 s32 $24 \quad s 8326$ \\
\hline 137 & $A_{g}$ & 1311.59 & 0.00 & 314.42 & 1313.36 s35 $10 \quad$ s91 21 \\
\hline 138 & $A_{g}$ & 1320.22 & 0.00 & 141.44 & $1321.11 \quad s 6012$ \\
\hline 139 & $A_{u}$ & 1321.18 & 3.81 & 0.00 & $1330.13 \quad s 5013 \quad s 9024$ \\
\hline 140 & $A_{g}$ & 1339.83 & 0.00 & 67.33 & 1340.55 s36 16 s73 -11 s82 $-12 \quad s 9013$ \\
\hline 141 & $A_{u}$ & 1340.57 & 4.11 & 0.00 & 1362.91 s74 12 s76 20 \\
\hline 142 & $A_{u}$ & 1366.86 & 19.42 & 0.00 & $1366.88 \quad s 74-17 \quad s 7610$ \\
\hline 143 & $A_{g}$ & 1367.34 & 0.00 & 245.65 & $1412.67 \quad s 7416 \quad s 7625$ \\
\hline 144 & $A_{g}$ & 1416.38 & 0.00 & 47.26 & $1416.53 \quad s 66-12 \quad s 7425 \quad s 76-16$ \\
\hline 145 & $A_{u}$ & 1416.98 & 51.83 & 0.00 & $1433.78 \quad s 2314 \quad s 2537 \quad s 26-37$ \\
\hline 146 & $A_{u}$ & 1433.77 & 416.37 & 0.00 & 1435.64 s24 -14 s25 -36 s26-36 \\
\hline 147 & $A_{g}$ & 1435.64 & 0.00 & 86.94 & 1451.99 s50 $11 \quad s 90-18$ \\
\hline 148 & $A_{g}$ & 1456.55 & 0.00 & 1165.72 & $1456.82 \quad s 73-39$ s118 16 \\
\hline 149 & $A_{u}$ & 1457.10 & 65.17 & 0.00 & 1466.81 s87 26 \\
\hline 150 & $A_{u}$ & 1467.60 & 84.44 & 0.00 & 1467.51 s80 31 \\
\hline 151 & $A_{g}$ & 1467.90 & 0.00 & 43.97 & 1485.18 s83 -19 s91 12 \\
\hline 152 & $A_{u}$ & 1485.31 & 45.63 & 0.00 & $1485.64 \quad s 35-10 \quad s 8313$ s91 20 \\
\hline
\end{tabular}




\begin{tabular}{|c|c|c|c|c|c|}
\hline 153 & $A_{g}$ & 1485.68 & 0.00 & 958.40 & 1491.62 \\
\hline 154 & $A_{g}$ & 1497.45 & 0.00 & 19.18 & 1497.49 s82 20 \\
\hline 155 & $A_{u}$ & 1497.64 & 115.89 & 0.00 & $1511.42 \quad s 8938 \quad s 9210$ \\
\hline 156 & $A_{g}$ & 1519.64 & 0.00 & 1024.55 & $1520.02 \quad s 84-26$ s118 19 \\
\hline 157 & $A_{u}$ & 1520.96 & 75.46 & 0.00 & $1540.96 \quad$ s59 $-20 \quad$ s123 17 \\
\hline 158 & $A_{g}$ & 1544.27 & 0.00 & 5052.34 & $1544.42 \quad s 42-16 \quad s 5827$ \\
\hline 159 & $A_{u}$ & 1544.62 & 46.54 & 0.00 & 1551.84 s46 -28 s55 20 \\
\hline 160 & $A_{u}$ & 1567.48 & 33.66 & 0.00 & 1567.49 s42 $11 \quad$ s52 $-12 \quad$ s54 $20 \quad$ s124 12 \\
\hline 161 & $A_{g}$ & 1567.58 & 0.00 & 449.16 & 1599.59 s30 $12 \quad$ s38 $-30 \quad$ s82 -12 \\
\hline 162 & $A_{g}$ & 1610.40 & 0.00 & 288.91 & 1610.40 \\
\hline 163 & $A_{u}$ & 1610.41 & 34.93 & 0.00 & 1610.41 s29 11 s34 22 s39 14 \\
\hline 164 & $A_{g}$ & 1613.42 & 0.00 & 54.15 & 1610.42 s31 $10 \quad$ s34 -18 s39 19 \\
\hline 165 & $A_{u}$ & 1614.67 & 18.23 & 0.00 & 1614.49 s41 -37 s88 -11 \\
\hline 166 & $A_{u}$ & 1631.57 & 131.59 & 0.00 & $1631.53 \quad s 4325 \quad s 47-24$ \\
\hline 167 & $A_{g}$ & 1631.78 & 0.00 & 1745.96 & $1631.77 \quad s 4324 \quad s 4725$ \\
\hline 168 & $A_{g}$ & 1647.09 & 0.00 & 968.99 & 1636.88 s37-44 \\
\hline 169 & $A_{u}$ & 1647.39 & 37.49 & 0.00 & $1647.27 \quad s 4039 \quad s 92-12$ \\
\hline 170 & $A_{g}$ & 1719.98 & 0.00 & 28.51 & $1715.76 \quad s 7527 \quad s 7735 \quad s 13211 \quad s 134-14$ \\
\hline 171 & $A_{u}$ & 1719.99 & 46.92 & 0.00 & $1719.97 \quad s 7534 \quad s 77-28 \quad s 13214 \quad s 13412$ \\
\hline 172 & $A_{u}$ & 2297.98 & 2928.53 & 0.00 & 2297.99 s21-33 s22 33 s23 12 s25-10 $\quad$ s26 10 \\
\hline 173 & $A_{g}$ & 2303.21 & 0.00 & 182.26 & 2303.22 s21 36 s22 36 \\
\hline 174 & $A_{u}$ & 2335.20 & 1268.89 & 0.00 & 2335.22 s23 66 \\
\hline 175 & $A_{g}$ & 2339.10 & 0.00 & 1215.14 & 2339.11 s24 71 \\
\hline 176 & $A_{u}$ & 3171.51 & 34.00 & 0.00 & 3171.13 s5 -31 s6 -13 s13 -38 s14 -11 \\
\hline 177 & $A_{g}$ & 3171.52 & 0.00 & 268.32 & 3171.53 s5 -33 s6 -12 s13 38 s14 10 \\
\hline 178 & $A_{g}$ & 3190.43 & 0.00 & 208.62 & 3190.17 s5 10 s6 -34 s14 -33 \\
\hline 179 & $A_{u}$ & 3190.43 & 7.69 & 0.00 & 3190.45 s6 $34 \quad$ s14 -35 \\
\hline 180 & $A_{u}$ & 3192.34 & 15.26 & 0.00 & $3192.34 \quad$ s10 74 \\
\hline 181 & $A_{g}$ & 3192.38 & 0.00 & 205.28 & 3192.36 s18 74 \\
\hline 182 & $A_{g}$ & 3206.27 & 0.00 & 271.46 & 3206.11 s9 -56 s17 -35 \\
\hline 183 & $A_{u}$ & 3206.42 & 34.02 & 0.00 & 3206.44 s9 35 s17 -57 \\
\hline 184 & $A_{u}$ & 3208.94 & 15.48 & 0.00 & 3208.79 s11 40 s15 -36 \\
\hline 185 & $A_{g}$ & 3208.95 & 0.00 & 599.67 & 3208.97 s11 -39 s15 -36 \\
\hline 186 & $A_{g}$ & 3215.82 & 0.00 & 410.55 & 3214.70 s12 81 \\
\hline 187 & $A_{u}$ & 3215.96 & 67.94 & 0.00 & 3215.85 s20 81 \\
\hline 188 & $A_{u}$ & 3223.73 & 1.14 & 0.00 & 3223.13 s7 39 s19 40 \\
\hline 189 & $A_{g}$ & 3223.73 & 0.00 & 165.61 & 3223.75 s7 44 s19 -35 \\
\hline 190 & $A_{g}$ & 3233.32 & 0.00 & 195.12 & $3233.24 \quad s 8-15 \quad s 1673$ \\
\hline 191 & $A_{u}$ & 3233.32 & 5.72 & 0.00 & $3233.30 \quad$ s8 $73 \quad$ s16 14 \\
\hline 192 & $A_{u}$ & 3416.74 & 210.09 & 0.00 & 3388.94 s1 -24 s2 -21 s3 -23 s4 -22 \\
\hline 193 & $A_{g}$ & 3416.82 & 0.00 & 312.31 & 3416.76 s2 -49 s4 46 \\
\hline 194 & $A_{g}$ & 3494.75 & 0.00 & 133.44 & 3423.70 s1 22 s2 -26 s3 22 s4 -28 \\
\hline 195 & $A_{u}$ & 3494.76 & 111.64 & 0.00 & 3494.78 s1 48 s3 -48 \\
\hline
\end{tabular}


Table S5. Description of the local modes in internal coordinates and calculated frequencies $f$, as analyzed by the VEDA program [S1] for the LS Fe(II) complex $\left[\mathrm{Fe}\left\{\mathrm{N}(\mathrm{CN})_{2}\right\}_{2}(\mathrm{abpt})_{2}\right]$ (atoms' numbering as in Table S3 and Figure S3).

\begin{tabular}{|c|c|c|c|c|c|c|c|c|c|c|c|c|}
\hline \multirow[t]{2}{*}{ s1 } & 1.00 & STRE & 11 & 12 & $\mathrm{NH}$ & 1.018280 & f3495 & 48 & f3424 & 22 & f3389 & 24 \\
\hline & -1.00 & & 11 & 13 & $\mathrm{NH}$ & 1.020259 & & & & & & \\
\hline \multirow[t]{2}{*}{ s2 } & 1.00 & STRE & 11 & 12 & $\mathrm{NH}$ & 1.018280 & f3424 & 26 & f3417 & 49 & f3389 & 21 \\
\hline & 1.00 & & 11 & 13 & $\mathrm{NH}$ & 1.020259 & & & & & & \\
\hline \multirow[t]{2}{*}{ s3 } & 1.00 & STRE & 44 & 45 & $\mathrm{NH}$ & 1.018280 & f3495 & 48 & f3424 & 22 & f3389 & 23 \\
\hline & -1.00 & & 44 & 46 & $\mathrm{NH}$ & 1.020259 & & & & & & \\
\hline \multirow[t]{2}{*}{ s 4} & 1.00 & STRE & 44 & 45 & $\mathrm{NH}$ & 1.018280 & f3424 & 28 & f3417 & 46 & f3389 & 22 \\
\hline & 1.00 & & 44 & 46 & $\mathrm{NH}$ & 1.020259 & & & & & & \\
\hline \multirow[t]{3}{*}{ s5 } & 1.00 & STRE & 18 & 19 & $\mathrm{CH}$ & 1.081786 & f3190 & 10 & f3172 & 33 & f3171 & 31 \\
\hline & -1.00 & & 21 & 22 & $\mathrm{CH}$ & 1.082698 & & & & & & \\
\hline & -1.00 & & 30 & 31 & $\mathrm{CH}$ & 1.084220 & & & & & & \\
\hline s 6 & -1.00 & STRE & 18 & 19 & $\mathrm{CH}$ & 1.081786 & f3190 & 34 & f3190 & 34 & f3172 & 12 \\
\hline \multirow[t]{3}{*}{ f3171 } & 13 & & & & & & & & & & & \\
\hline & 1.00 & & 21 & 22 & $\mathrm{CH}$ & 1.082698 & & & & & & \\
\hline & -1.00 & & 30 & 31 & $\mathrm{CH}$ & 1.084220 & & & & & & \\
\hline \multirow[t]{3}{*}{ s 7} & 1.00 & STRE & 15 & 16 & $\mathrm{CH}$ & 1.080516 & f3224 & 44 & f3223 & 39 & & \\
\hline & 1.00 & & 21 & 22 & $\mathrm{CH}$ & 1.082698 & & & & & & \\
\hline & 1.00 & & 30 & 31 & $\mathrm{CH}$ & 1.084220 & & & & & & \\
\hline s 8 & 1.00 & STRE & 26 & 27 & $\mathrm{CH}$ & 1.079474 & f3233 & 73 & f3233 & 15 & & \\
\hline \multirow[t]{3}{*}{ s9 } & -1.00 & STRE & 24 & 25 & $\mathrm{CH}$ & 1.082448 & f3206 & 35 & f3206 & 56 & & \\
\hline & 1.00 & & 28 & 29 & $\mathrm{CH}$ & 1.081073 & & & & & & \\
\hline & -1.00 & & 32 & 33 & $\mathrm{CH}$ & 1.081559 & & & & & & \\
\hline \multirow{4}{*}{ s10 } & 1.00 & STRE & 24 & 25 & $\mathrm{CH}$ & 1.082448 & f3192 & 74 & & & & \\
\hline & -1.00 & & 26 & 27 & $\mathrm{CH}$ & 1.079474 & & & & & & \\
\hline & 1.00 & & 28 & 29 & $\mathrm{CH}$ & 1.081073 & & & & & & \\
\hline & -1.00 & & 32 & 33 & $\mathrm{CH}$ & 1.081559 & & & & & & \\
\hline \multirow[t]{3}{*}{ s 11} & 1.00 & STRE & 18 & 19 & $\mathrm{CH}$ & 1.081786 & f3209 & 39 & f3209 & 40 & & \\
\hline & 1.00 & & 21 & 22 & $\mathrm{CH}$ & 1.082698 & & & & & & \\
\hline & 1.00 & & 30 & 31 & $\mathrm{CH}$ & 1.084220 & & & & & & \\
\hline \multirow[t]{4}{*}{ s 12} & 1.00 & STRE & 24 & 25 & $\mathrm{CH}$ & 1.082448 & f3215 & 81 & & & & \\
\hline & 1.00 & & 26 & 27 & $\mathrm{CH}$ & 1.079474 & & & & & & \\
\hline & 1.00 & & 28 & 29 & $\mathrm{CH}$ & 1.081073 & & & & & & \\
\hline & 1.00 & & 32 & 33 & $\mathrm{CH}$ & 1.081559 & & & & & & \\
\hline \multirow[t]{4}{*}{ s13 } & 1.00 & STRE & 48 & 49 & $\mathrm{CH}$ & 1.080516 & f3172 & 38 & f3171 & 38 & & \\
\hline & 1.00 & & 51 & 52 & $\mathrm{CH}$ & 1.081786 & & & & & & \\
\hline & -1.00 & & 54 & 55 & $\mathrm{CH}$ & 1.082698 & & & & & & \\
\hline & -1.00 & & 63 & 64 & $\mathrm{CH}$ & 1.084220 & & & & & & \\
\hline s14 & -1.00 & STRE & 48 & 49 & $\mathrm{CH}$ & 1.080516 & f3190 & 35 & f3190 & 33 & f3172 & 10 \\
\hline f3171 & 11 & & & & & & & & & & & \\
\hline & -1.00 & & 51 & 52 & $\mathrm{CH}$ & 1.081786 & & & & & & \\
\hline & 1.00 & & 54 & 55 & $\mathrm{CH}$ & 1.082698 & & & & & & \\
\hline & -1.00 & & 63 & 64 & $\mathrm{CH}$ & 1.084220 & & & & & & \\
\hline s15 & 1.00 & STRE & 48 & 49 & $\mathrm{CH}$ & 1.080516 & f3209 & 36 & f3209 & 36 & & \\
\hline & -1.00 & & 51 & 52 & $\mathrm{CH}$ & 1.081786 & & & & & & \\
\hline & -1.00 & & 54 & 55 & $\mathrm{CH}$ & 1.082698 & & & & & & \\
\hline & -1.00 & & 63 & 64 & $\mathrm{CH}$ & 1.084220 & & & & & & \\
\hline s16 & 1.00 & STRE & 59 & 60 & $\mathrm{CH}$ & 1.079474 & f3233 & 14 & f3233 & 73 & & \\
\hline s17 & -1.00 & STRE & 57 & 58 & $\mathrm{CH}$ & 1.082448 & f3206 & 57 & f3206 & 35 & & \\
\hline & 1.00 & & 61 & 62 & $\mathrm{CH}$ & 1.081073 & & & & & & \\
\hline & -1.00 & & 65 & 66 & $\mathrm{CH}$ & 1.081559 & & & & & & \\
\hline s18 & 1.00 & STRE & 57 & 58 & $\mathrm{CH}$ & 1.082448 & f3192 & 74 & & & & \\
\hline & -1.00 & & 59 & 60 & $\mathrm{CH}$ & 1.079474 & & & & & & \\
\hline & 1.00 & & 61 & 62 & $\mathrm{CH}$ & 1.081073 & & & & & & \\
\hline & -1.00 & & 65 & 66 & $\mathrm{CH}$ & 1.081559 & & & & & & \\
\hline s19 & 1.00 & STRE & 48 & 49 & $\mathrm{CH}$ & 1.080516 & f3224 & 35 & f3223 & 40 & & \\
\hline & 1.00 & & 51 & 52 & $\mathrm{CH}$ & 1.081786 & & & & & & \\
\hline & 1.00 & & 54 & 55 & $\mathrm{CH}$ & 1.082698 & & & & & & \\
\hline & 1.00 & & 63 & 64 & $\mathrm{CH}$ & 1.084220 & & & & & & \\
\hline s20 & 1.00 & STRE & 57 & 58 & $\mathrm{CH}$ & 1.082448 & f3216 & 81 & & & & \\
\hline & 1.00 & & 59 & 60 & $\mathrm{CH}$ & 1.079474 & & & & & & \\
\hline & 1.00 & & 61 & 62 & $\mathrm{CH}$ & 1.081073 & & & & & & \\
\hline
\end{tabular}




\begin{tabular}{|c|c|c|c|c|c|c|c|c|c|c|c|c|}
\hline & 1.00 & & 65 & 66 & $\mathrm{CH}$ & 1.081559 & & & & & & \\
\hline s21 & 1.00 & STRE & 34 & 14 & $\mathrm{NC}$ & 1.164081 & f2303 & 36 & f2298 & 33 & & \\
\hline s22 & 1.00 & STRE & 67 & 47 & $\mathrm{NC}$ & 1.164081 & f2303 & 36 & f2298 & 33 & & \\
\hline \multirow[t]{2}{*}{ s23 } & 1.00 & STRE & 8 & 23 & $\mathrm{NC}$ & 1.169864 & f2335 & 66 & f2298 & 12 & f1434 & 14 \\
\hline & -1.00 & & 41 & 56 & $\mathrm{NC}$ & 1.169864 & & & & & & \\
\hline \multirow{2}{*}{\multicolumn{2}{|c|}{$\mathrm{s} 241.00^{1.00}$}} & STRE & 8 & 23 & $\mathrm{NC}$ & 1.169864 & f2339 & 71 & f1436 & 14 & & \\
\hline & & 41 & 56 & $\mathrm{NC}$ & & 69864 & & & & & & \\
\hline \multirow[t]{2}{*}{$s 25$} & -1.00 & STRE & 20 & 14 & $\mathrm{NC}$ & 1.313753 & f2298 & 10 & f1436 & 36 & f1434 & 37 \\
\hline & 1.00 & & 20 & 23 & $\mathrm{NC}$ & 1.277781 & & & & & & \\
\hline \multirow[t]{2}{*}{ s 26} & -1.00 & STRE & 53 & 47 & $\mathrm{NC}$ & 1.313753 & f2298 & 10 & f1436 & 36 & f1434 & 37 \\
\hline & 1.00 & & 53 & 56 & $\mathrm{NC}$ & 1.277781 & & & & & & \\
\hline \multirow[t]{2}{*}{ s27 } & 1.00 & STRE & 20 & 14 & $\mathrm{NC}$ & 1.313753 & f939 & 38 & f938 38 & & & \\
\hline & 1.00 & & 20 & 23 & $\mathrm{NC}$ & 1.277781 & & & & & & \\
\hline \multirow[t]{2}{*}{$s 28$} & 1.00 & STRE & 53 & 47 & $\mathrm{NC}$ & 1.313753 & f939 & 38 & f938 38 & & & \\
\hline & 1.00 & & 53 & 56 & $\mathrm{NC}$ & 1.277781 & & & & & & \\
\hline \multirow[t]{3}{*}{ s29 } & -1.00 & STRE & 3 & 7 & $\mathrm{NC}$ & 1.342102 & f1610 & 11 & f1295 & 12 & f1294 & 44 \\
\hline & 1.00 & & 3 & 30 & $\mathrm{NC}$ & 1.333463 & & & & & & \\
\hline & 1.00 & & 10 & 9 & $\mathrm{NC}$ & 1.317852 & & & & & & \\
\hline \multirow[t]{4}{*}{ s30 } & -1.00 & STRE & 5 & 17 & $\mathrm{NC}$ & 1.355173 & $\mathrm{f} 1600$ & 12 & f1291 & 14 & & \\
\hline & 1.00 & & 5 & 28 & $\mathrm{NC}$ & 1.333794 & & & & & & \\
\hline & 1.00 & & 35 & 39 & $\mathrm{NC}$ & 1.320595 & & & & & & \\
\hline & -1.00 & & 38 & 61 & $\mathrm{NC}$ & 1.333794 & & & & & & \\
\hline s31 & -1.00 & STRE & 36 & 40 & $\mathrm{NC}$ & 1.342102 & f1610 & 10 & f1295 & 33 & f1294 & 11 \\
\hline \multirow{2}{*}{ f1291 } & 13 & & & & & & & & & & & \\
\hline & 1.00 & & 36 & 63 & $\mathrm{NC}$ & 1.333463 & & & & & & \\
\hline s32 & 1.00 & STRE & 3 & 7 & $\mathrm{NC}$ & 1.342102 & f1311 & 24 & & & & \\
\hline & 1.00 & & 3 & 30 & $\mathrm{NC}$ & 1.333463 & & & & & & \\
\hline & -1.00 & & 10 & 9 & $\mathrm{NC}$ & 1.317852 & & & & & & \\
\hline v33 & 1.00 & STRE & 1 & 5 & FeN & 2.047215 & f286 & 17 & f181 3 & 37 & & \\
\hline s34 & 1.00 & STRE & 15 & 21 & $\mathrm{CC}$ & 1.385665 & f1610 & 18 & f1610 & 22 & f1294 & 16 \\
\hline $\mathrm{f} 1184$ & 17 & & & & & & & & & & & \\
\hline & -1.00 & & 18 & 21 & $\mathrm{CC}$ & 1.390882 & & & & & & \\
\hline & 1.00 & & 18 & 30 & $\mathrm{CC}$ & 1.389323 & & & & & & \\
\hline s35 & 1.00 & STRE & 36 & 63 & $\mathrm{NC}$ & 1.333463 & f1486 & 10 & f1313 & 10 & f1309 & 25 \\
\hline & -1.00 & & 43 & 42 & $\mathrm{NC}$ & 1.317852 & & & & & & \\
\hline s36 & -1.00 & STRE & 5 & 17 & $\mathrm{NC}$ & 1.355173 & f1341 & 16 & & & & \\
\hline & -1.00 & & 5 & 28 & $\mathrm{NC}$ & 1.333794 & & & & & & \\
\hline & 1.00 & & 35 & 39 & $\mathrm{NC}$ & 1.320595 & & & & & & \\
\hline & 1.00 & & 38 & 61 & $\mathrm{NC}$ & 1.333794 & & & & & & \\
\hline s37 & -1.00 & STRE & 17 & 26 & $\mathrm{CC}$ & 1.390482 & f1637 & 44 & & & & \\
\hline & 1.00 & & 24 & 32 & $\mathrm{CC}$ & 1.386484 & & & & & & \\
\hline s38 & -1.00 & STRE & 24 & 26 & $\mathrm{CC}$ & 1.388708 & f1600 & 30 & $\mathrm{f} 1182$ & 11 & & \\
\hline & 1.00 & & 24 & 32 & $\mathrm{CC}$ & 1.386484 & & & & & & \\
\hline s39 & 1.00 & STRE & 48 & 54 & $\mathrm{CC}$ & 1.385665 & f1610 & 19 & f1610 & 14 & f1295 & 15 \\
\hline f1184 & 18 & & & & & & & & & & & \\
\hline & -1.00 & & 51 & 54 & $\mathrm{CC}$ & 1.390882 & & & & & & \\
\hline & 1.00 & & 51 & 63 & $\mathrm{CC}$ & 1.389323 & & & & & & \\
\hline s 40 & -1.00 & STRE & 50 & 59 & $\mathrm{CC}$ & 1.390482 & f1647 & 39 & f1291 & 11 & & \\
\hline & 1.00 & & 57 & 59 & $\mathrm{CC}$ & 1.388708 & & & & & & \\
\hline & 1.00 & & 57 & 65 & $\mathrm{CC}$ & 1.386484 & & & & & & \\
\hline s41 & -1.00 & STRE & 50 & 59 & $\mathrm{CC}$ & 1.390482 & f1614 & 37 & & & & \\
\hline & -1.00 & & 57 & 59 & $\mathrm{CC}$ & 1.388708 & & & & & & \\
\hline & 1.00 & & 57 & 65 & $\mathrm{CC}$ & 1.386484 & & & & & & \\
\hline s 42 & 1.00 & STRE & 3 & 7 & $\mathrm{NC}$ & 1.342102 & f1567 & 11 & f1544 & 16 & & \\
\hline & 1.00 & & 3 & 30 & $\mathrm{NC}$ & 1.333463 & & & & & & \\
\hline & 1.00 & & 10 & 9 & $\mathrm{NC}$ & 1.317852 & & & & & & \\
\hline s43 & -1.00 & STRE & 15 & 21 & $\mathrm{CC}$ & 1.385665 & f1632 & 24 & f1632 & 25 & f1124 & 25 \\
\hline & 1.00 & & 18 & 30 & $\mathrm{CC}$ & 1.389323 & & & & & & \\
\hline s 44 & 1.00 & STRE & 15 & 21 & $\mathrm{CC}$ & 1.385665 & f1066 & 50 & & & & \\
\hline & 1.00 & & 18 & 21 & $\mathrm{CC}$ & 1.390882 & & & & & & \\
\hline & 1.00 & & 18 & 30 & $\mathrm{CC}$ & 1.389323 & & & & & & \\
\hline s 45 & 1.00 & STRE & 24 & 26 & $\mathrm{CC}$ & 1.388708 & f1106 & 18 & f1094 & 10 & f1086 & 13 \\
\hline & 1.00 & & 24 & 32 & $\mathrm{CC}$ & 1.386484 & & & & & & \\
\hline s 46 & 1.00 & STRE & 36 & 40 & $\mathrm{NC}$ & 1.342102 & f1552 & 28 & & & & \\
\hline & 1.00 & & 43 & 42 & $\mathrm{NC}$ & 1.317852 & & & & & & \\
\hline s 47 & -1.00 & STRE & 48 & 54 & $\mathrm{CC}$ & 1.385665 & f1632 & 25 & f1632 & 24 & f1124 & 22 \\
\hline & 1.00 & & 51 & 63 & $\mathrm{CC}$ & 1.389323 & & & & & & \\
\hline s48 & 1.00 & STRE & 48 & 54 & $\mathrm{CC}$ & 1.385665 & f1070 & 26 & f1061 & 14 & f1018 & 16 \\
\hline
\end{tabular}




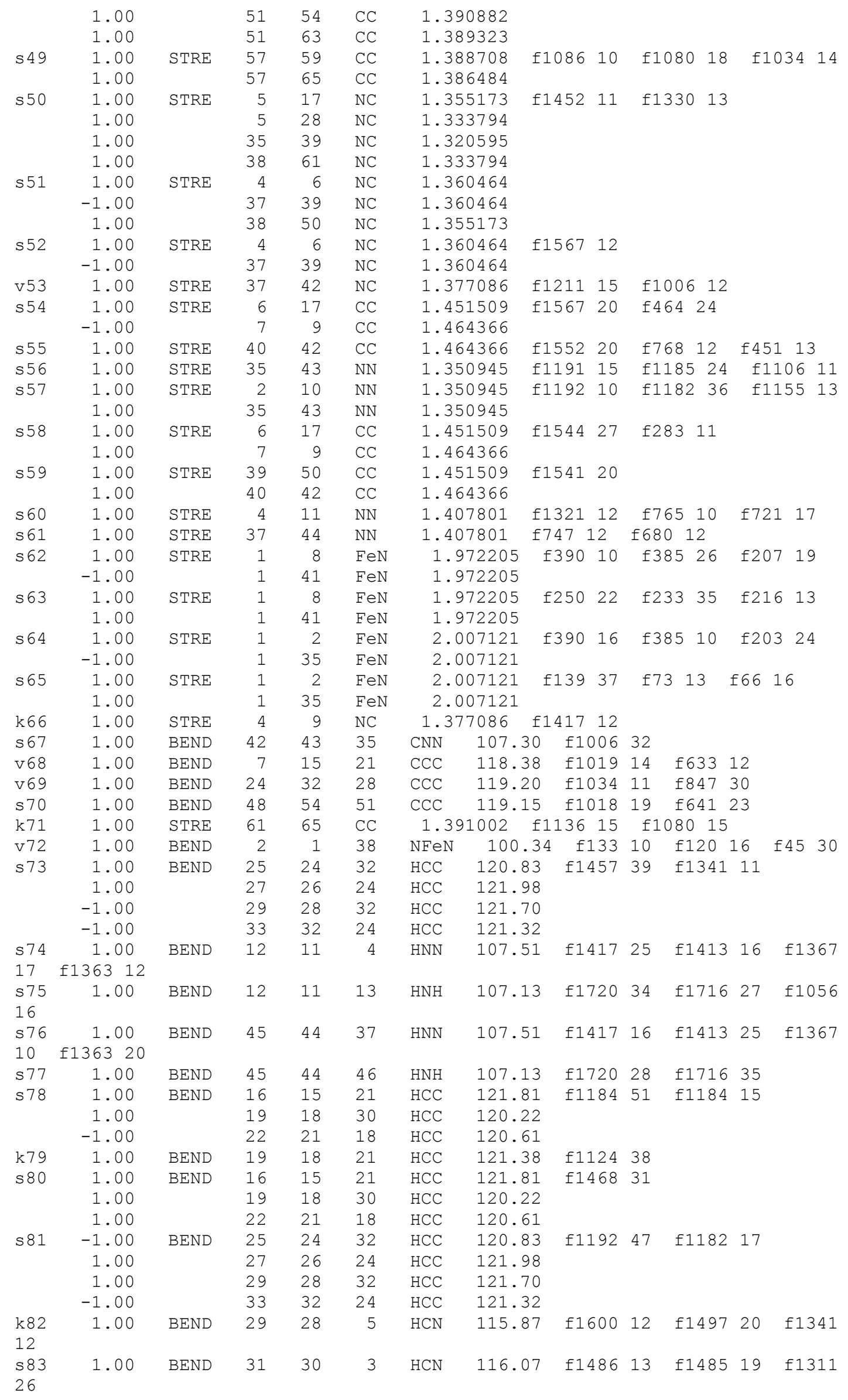




\begin{tabular}{|c|c|c|c|c|c|c|c|c|c|c|c|c|}
\hline \multirow{2}{*}{$\begin{array}{l}\text { s84 } \\
17\end{array}$} & 1.00 & BEND & 27 & 26 & 24 & $\mathrm{HCC}$ & 121.98 & f1520 & 26 & f1106 & 14 & f1094 \\
\hline & 1.00 & & 29 & 28 & 32 & $\mathrm{HCC}$ & 121.70 & & & & & \\
\hline & 1.00 & & 33 & 32 & 24 & $\mathrm{HCC}$ & 121.32 & & & & & \\
\hline \multirow[t]{3}{*}{ s 85} & 1.00 & BEND & 49 & 48 & 54 & $\mathrm{HCC}$ & 121.81 & $\mathrm{f} 1184$ & 14 & f1184 & 51 & \\
\hline & 1.00 & & 52 & 51 & 63 & $\mathrm{HCC}$ & 120.22 & & & & & \\
\hline & -1.00 & & 55 & 54 & 51 & $\mathrm{HCC}$ & 120.61 & & & & & \\
\hline $\mathrm{k} 86$ & 1.00 & BEND & 52 & 51 & 54 & $\mathrm{HCC}$ & 121.38 & f1124 & 31 & & & \\
\hline \multirow[t]{3}{*}{ s 87} & 1.00 & BEND & 49 & 48 & 54 & $\mathrm{HCC}$ & 121.81 & f1467 & 26 & f1070 & 10 & \\
\hline & 1.00 & & 52 & 51 & 63 & $\mathrm{HCC}$ & 120.22 & & & & & \\
\hline & 1.00 & & 55 & 54 & 51 & $\mathrm{HCC}$ & 120.61 & & & & & \\
\hline k88 & 1.00 & BEND & 58 & 57 & 59 & $\mathrm{HCC}$ & 120.17 & f1614 & 11 & f1191 & 14 & $\mathrm{f} 1185$ \\
\hline 18 & $f 113611$ & & & & & & & & & & & \\
\hline \multirow[t]{3}{*}{ s89 } & 1.00 & BEND & 60 & 59 & 57 & $\mathrm{HCC}$ & 121.98 & f1511 & -38 & & & \\
\hline & 1.00 & & 62 & 61 & 65 & $\mathrm{HCC}$ & 121.70 & & & & & \\
\hline & -1.00 & & 66 & 65 & 61 & $\mathrm{HCC}$ & 119.48 & & & & & \\
\hline \multirow{3}{*}{$\begin{array}{l}\text { s90 } \\
24\end{array}$} & 1.00 & BEND & 58 & 57 & 65 & $\mathrm{HCC}$ & 120.83 & f1452 & 18 & f1341 & 13 & f1330 \\
\hline & 1.00 & & 60 & 59 & 57 & $\mathrm{HCC}$ & 121.98 & & & & & \\
\hline & 1.00 & & 66 & 65 & 61 & $\mathrm{HCC}$ & 119.48 & & & & & \\
\hline \multirow{2}{*}{$\begin{array}{l}\text { s91 } \\
21\end{array}$} & 1.00 & BEND & 64 & 63 & 36 & $\mathrm{HCN}$ & 116.07 & f1486 & 520 & f1485 & 12 & f1313 \\
\hline & & & & & & & & & & & & \\
\hline \multirow{2}{*}{$\begin{array}{l}\text { k92 } \\
15\end{array}$} & 1.00 & BEND & 66 & 65 & 57 & $\mathrm{HCC}$ & 121.32 & f1647 & 12 & f1511 & 10 & f1191 \\
\hline & $f 113616$ & & & & & & & & & & & \\
\hline \multirow{2}{*}{$\begin{array}{l}\text { s93 } \\
\text { f519 }\end{array}$} & 1.00 & BEND & 20 & 14 & 34 & $\mathrm{NCN}$ & 175.61 & f65 6 & 14 & f65615 & & $f 52330$ \\
\hline & 12 & & & & & & & & & & & \\
\hline \multirow{3}{*}{$\begin{array}{l}\text { s94 } \\
\text { s95 }\end{array}$} & 1.00 & BEND & 53 & 47 & 67 & $\mathrm{NCN}$ & 175.61 & f656 & 14 & f656 12 & & $f 52241$ \\
\hline & 1.00 & BEND & 23 & 8 & 1 & $\mathrm{CNFe}$ & 177.09 & f28 & 14 & f15 65 & & \\
\hline & -1.00 & & 56 & 41 & 1 & $\mathrm{CNFe}$ & 177.09 & & & & & \\
\hline \multirow[t]{2}{*}{ s96 } & 1.00 & BEND & 23 & 8 & 1 & $\mathrm{CNFe}$ & 177.09 & f45 & 17 & f10 39 & & \\
\hline & 1.00 & & 56 & 41 & 1 & $\mathrm{CNFe}$ & 177.09 & & & & & \\
\hline s97 & 1.00 & BEND & 8 & 23 & 20 & $\mathrm{NCN}$ & 174.16 & f65 6 & 20 & $f 65621$ & & f523 20 \\
\hline f519 & 10 & & & & & & & & & & & \\
\hline s98 & 1.00 & BEND & 41 & 56 & 53 & $\mathrm{NCN}$ & 174.16 & f656 & 21 & f65618 & & f522 28 \\
\hline s99 & 1.00 & BEND & 14 & 20 & 23 & $\mathrm{CNC}$ & 124.65 & f65 6 & 12 & $f 65613$ & & f95 10 \\
\hline s100 & 1.00 & BEND & 47 & 53 & 56 & $\mathrm{CNC}$ & 124.65 & f65 6 & 12 & $f 65611$ & & f95 10 \\
\hline s101 & 1.00 & BEND & 39 & 35 & 1 & $\mathrm{CNFe}$ & 113.93 & f451 & -18 & f374 1 & & \\
\hline s102 & 1.00 & BEND & 7 & 3 & 30 & $\mathrm{CNC}$ & 118.16 & f654 & 10 & f633 13 & & \\
\hline v103 & 1.00 & BEND & 32 & 28 & 5 & $\mathrm{CCN}$ & 122.43 & f1155 & 510 & & & \\
\hline s104 & 1.00 & BEND & 40 & 36 & 63 & $\mathrm{CNC}$ & 118.16 & f203 & 13 & & & \\
\hline & -1.00 & & 42 & 40 & 36 & $\mathrm{CCN}$ & 117.17 & & & & & \\
\hline k105 & 1.00 & BEND & 61 & 38 & 1 & $\mathrm{CNFe}$ & 126.12 & f660 & 21 & & & \\
\hline s106 & 1.00 & BEND & 17 & 26 & 24 & $\mathrm{CCC}$ & 118.38 & f660 & 10 & f654 14 & & \\
\hline s107 & 1.00 & BEND & 59 & 57 & 65 & $\mathrm{CCC}$ & 119.00 & f1047 & 710 & $f 6603$ & 32 & \\
\hline s108 & 1.00 & BEND & 9 & 7 & 3 & $\mathrm{CCN}$ & 117.17 & f404 & 12 & & & \\
\hline s109 & 1.00 & BEND & 18 & 30 & 3 & $\mathrm{CCN}$ & 123.19 & f654 & 20 & & & \\
\hline s110 & 1.00 & BEND & 21 & 18 & 30 & $\mathrm{CCC}$ & 118.40 & f765 & 21 & & & \\
\hline k111 & 1.00 & BEND & 6 & 17 & 26 & $\mathrm{CCC}$ & 125.83 & f404 & 23 & & & \\
\hline s112 & 1.00 & BEND & 40 & 36 & 63 & $\mathrm{CNC}$ & 118.16 & f1061 & 13 & & & \\
\hline s113 & 1.00 & BEND & 51 & 63 & 36 & $\mathrm{CCN}$ & 123.19 & f1018 & 311 & $f 6412$ & 23 & \\
\hline s114 & 1.00 & BEND & 54 & 51 & 63 & $\mathrm{CCC}$ & 118.40 & f768 & 19 & & & \\
\hline s115 & 1.00 & BEND & 59 & 50 & 38 & $\mathrm{CCN}$ & 122.70 & & & & & \\
\hline s116 & 1.00 & BEND & 6 & 17 & 5 & $\mathrm{CCN}$ & 111.46 & f658 & 19 & & & \\
\hline & 1.00 & & 17 & 5 & 28 & $\mathrm{CNC}$ & 118.28 & & & & & \\
\hline & -1.00 & & 39 & 50 & 38 & $\mathrm{CCN}$ & 111.46 & & & & & \\
\hline & -1.00 & & 50 & 38 & 61 & $\mathrm{CNC}$ & 118.28 & & & & & \\
\hline k117 & 1.00 & BEND & 50 & 38 & 1 & $\mathrm{CNFe}$ & 115.59 & f 660 & 14 & & & \\
\hline k118 & 1.00 & BEND & 6 & 4 & 9 & $\mathrm{CNC}$ & 105.56 & f1520 & 19 & f1457 & 16 & \\
\hline k119 & 1.00 & BEND & 39 & 37 & 42 & $\mathrm{CNC}$ & 105.56 & & & & & \\
\hline s120 & 1.00 & BEND & 7 & 9 & 10 & $\mathrm{CCN}$ & 124.90 & & & & & \\
\hline s121 & -1.00 & BEND & 40 & 42 & 43 & $\mathrm{CCN}$ & 124.90 & & & & & \\
\hline & 1.00 & & 50 & 39 & 37 & $\mathrm{CCN}$ & 132.19 & & & & & \\
\hline s122 & 1.00 & BEND & 6 & 2 & 10 & CNN & 109.32 & f1014 & 12 & & & \\
\hline & -1.00 & & 39 & 35 & 43 & CNN & 109.32 & & & & & \\
\hline s123 & 1.00 & BEND & 39 & 35 & 43 & CNN & 109.32 & f1541 & -17 & & & \\
\hline k124 & 1.00 & BEND & 7 & 9 & 4 & $\mathrm{CCN}$ & 125.69 & f1567 & 12 & & & \\
\hline k125 & 1.00 & BEND & 40 & 42 & 37 & $\mathrm{CCN}$ & 125.69 & f680 & 13 & & & \\
\hline
\end{tabular}




\begin{tabular}{|c|c|c|c|c|c|c|c|c|c|c|c|}
\hline $\begin{array}{l}\mathrm{k} 126 \\
\mathrm{f} 320\end{array}$ & $28^{1.00}$ & BEND & 9 & 4 & 11 & CNN & 129.31 & \multicolumn{4}{|c|}{ f633 10} \\
\hline s127 & 1.00 & BEND & 42 & 37 & 44 & CNN & 129.31 & f342 1 & f334 10 & f322 & 26 \\
\hline $\mathrm{k} 128$ & 1.00 & BEND & 8 & 1 & 35 & $\mathrm{NF} e \mathrm{~N}$ & 90.23 & $23 \mp 385$ & f207 11 & 711 f9 18 & 18 \\
\hline k129 & 1.00 & BEND & 35 & 1 & 41 & $\mathrm{NF} e \mathrm{~N}$ & 89.77 & $\mathrm{f} 151$ & f10 11 & 11 & \\
\hline s130 & 1.00 & BEND & 38 & 1 & 41 & $\mathrm{NF} e \mathrm{~N}$ & 90.19 & f25 1 & 11 & & \\
\hline s131 & 1.00 & BEND & 10 & 2 & 1 & NNFe & \multicolumn{2}{|c|}{136.74 f633 } & 11 f45 & 15 & \\
\hline s132 & 1.00 & TORS & 12 & 11 & 4 & 9 & HNNC & -83.64 & f1720 14 & $4 \quad$ f1716 11 & 11 \\
\hline f1056 & 38 & & & & & & & & & & \\
\hline & -1.00 & & 13 & 11 & 4 & 9 & HNNC & 30.40 & & & \\
\hline s133 & 1.00 & TORS & 12 & 11 & 4 & 9 & HNNC & -83.64 & f283 12 & $\mathrm{f} 274 \quad 22$ & \\
\hline f266 4 & 40 & & & & & & & & & & \\
\hline & 1.00 & & 13 & 11 & 4 & 9 & HNNC & 30.40 & & & \\
\hline s134 & 1.00 & TORS & 45 & 44 & 37 & 39 & HNNC & -93.69 & $\mathrm{f} 1720 \quad 12$ & $\mathrm{f} 17161$ & 14 \\
\hline f1047 & 18 & & & & & & & & & & \\
\hline & -1.00 & & 46 & 44 & 37 & 39 & HNNC & -207.73 & & & \\
\hline k135 & 1.00 & TORS & 46 & 44 & 37 & 42 & HNNC & -30.40 & f283 11 & f268 47 & \\
\hline k136 & 1.00 & OUT & 15 & 7 & 21 & 16 & $\mathrm{CCCH}$ & 0.09 & f791 20 & $f 79125$ & \\
\hline k137 & 1.00 & TORS & 19 & 18 & 30 & 31 & $\mathrm{HCCH}$ & 0.03 & f1013 19 & $9 \quad f 98437$ & \\
\hline f982 3 & 33 & & & & & & & & & & \\
\hline k138 & 1.00 & TORS & 16 & 15 & 21 & 22 & $\mathrm{HCCH}$ & -0.11 & $\mathrm{f} 101363$ & & \\
\hline k139 & 1.00 & TORS & 25 & 24 & 32 & 33 & $\mathrm{HCCH}$ & -0.43 & f1009 38 & f1008 3 & \\
\hline s140 & 1.00 & TORS & 27 & 26 & 24 & 32 & $\mathrm{HCCC}$ & -180.59 & f925 34 & f925 34 & \\
\hline & 1.00 & & 29 & 28 & 32 & 24 & $\mathrm{HCCC}$ & -179.48 & & & \\
\hline & -1.00 & & 33 & 32 & 28 & 5 & $\mathrm{HCCN}$ & -179.89 & & & \\
\hline k141 & 1.00 & OUT & 28 & 32 & 5 & 29 & $\mathrm{CCNH}$ & 0.05 & f1001 38 & $3 \quad f 1000 \quad 40$ & \\
\hline s142 & 1.00 & TORS & 16 & 15 & 7 & 9 & $\mathrm{HCCC}$ & -1.73 & f916 42 & f911 36 & \\
\hline & 1.00 & & 19 & 18 & 30 & 3 & $\mathrm{HCCN}$ & -180.26 & & & \\
\hline & 1.00 & & 22 & 21 & 15 & 7 & $\mathrm{HCCC}$ & -179.84 & & & \\
\hline & 1.00 & & 31 & 30 & 3 & 7 & $\mathrm{HCNC}$ & -180.25 & & & \\
\hline s143 & 1.00 & TORS & 25 & 24 & 32 & 28 & $\mathrm{HCCC}$ & -180.69 & $f 5730 \quad 25$ & f784 42 & \\
\hline & 1.00 & & 27 & 26 & 24 & 32 & $\mathrm{HCCC}$ & -180.59 & & & \\
\hline & 1.00 & & 33 & 32 & 28 & 5 & $\mathrm{HCCN}$ & -179.89 & & & \\
\hline s144 & 1.00 & TORS & 49 & 48 & 54 & 51 & $\mathrm{HCCC}$ & -180.07 & f1014 16 & $\mathrm{f} 10135$ & 53 \\
\hline & 1.00 & & 52 & 51 & 63 & 36 & $\mathrm{HCCN}$ & -179.74 & & & \\
\hline & -1.00 & & 55 & 54 & 51 & 63 & $\mathrm{HCCC}$ & -180.23 & & & \\
\hline & -1.00 & & 64 & 63 & 36 & 40 & $\mathrm{HCNC}$ & -179.75 & & & \\
\hline k145 & 1.00 & OUT & 51 & 54 & 63 & 52 & $\mathrm{CCCH}$ & 0.00 & f791 28 & f791 21 & \\
\hline s146 & 1.00 & TORS & 49 & 48 & 54 & 51 & $\mathrm{HCCC}$ & -180.07 & f916 41 & f911 29 & \\
\hline & -1.00 & & 52 & 51 & 63 & 36 & $\mathrm{HCCN}$ & -179.74 & & & \\
\hline & 1.00 & & 55 & 54 & 51 & 63 & $\mathrm{HCCC}$ & -180.23 & & & \\
\hline & -1.00 & & 64 & 63 & 36 & 40 & $\mathrm{HCNC}$ & -179.75 & & & \\
\hline k147 & 1.00 & TORS & 58 & 57 & 59 & 50 & $\mathrm{HCCC}$ & -180.20 & $f 5730 \quad 20$ & f784 30 & \\
\hline s148 & 1.00 & TORS & 60 & 59 & 57 & 65 & $\mathrm{HCCC}$ & -179.41 & f925 41 & f925 39 & \\
\hline & 1.00 & & 62 & 61 & 65 & 57 & $\mathrm{HCCC}$ & -180.52 & & & \\
\hline & -1.00 & & 66 & 65 & 61 & 38 & $\mathrm{HCCN}$ & -180.11 & & & \\
\hline s149 & 1.00 & TORS & 58 & 57 & 65 & 61 & $\mathrm{HCCC}$ & -179.31 & f1001 35 & $\mathrm{f} 10003$ & 32 \\
\hline & -1.00 & & 60 & 59 & 57 & 65 & $\mathrm{HCCC}$ & -179.41 & & & \\
\hline & 1.00 & & 62 & 61 & 65 & 57 & $\mathrm{HCCC}$ & -180.52 & & & \\
\hline k150 & 1.00 & TORS & 52 & 51 & 63 & 64 & $\mathrm{HCCH}$ & -0.03 & f984 37 & f982 37 & \\
\hline k151 & 1.00 & TORS & 62 & 61 & 65 & 66 & $\mathrm{HCCH}$ & -0.2 & $5 £ 1009$ & $31 \mathrm{f1008}$ & 827 \\
\hline f1001 & 10 & & & & & & & & & & \\
\hline & & & & & & & & & .00013 & & \\
\hline s152 & 1.00 & TORS & 23 & 20 & 14 & 34 & $\mathrm{CNCN}$ & -180.34 & $f 528 \quad 10$ & $\mathrm{f} 528 \quad 78$ & \\
\hline s153 & 1.00 & TORS & 56 & 53 & 47 & 67 & $\mathrm{CNCN}$ & -179.66 & f528 75 & f528 12 & \\
\hline k154 & 1.00 & TORS & 23 & 8 & 1 & 35 & CNFeN & -22.46 & f5 19 & f4 27 & \\
\hline $\begin{array}{l}\mathrm{k} 155 \\
15\end{array}$ & 1.00 & TORS & 56 & 41 & 1 & 35 & $\mathrm{CNF} e \mathrm{~N}$ & -157.54 & f28 11 & f5 31 & $\mathrm{f} 4$ \\
\hline $\begin{array}{l}15 \\
\text { s156 }\end{array}$ & 1.00 & TORS & 20 & 23 & 8 & 1 & $\mathrm{NCNFe}$ & -153.50 & $\mathrm{f} 28 \quad 20$ & f25 14 & f5 \\
\hline 10 f 4 & 17 & & & & & & & & & & \\
\hline s157 & 1.00 & TORS & 53 & 56 & 41 & 1 & $\mathrm{NCNFe}$ & -206.50 & f28 23 & $\mathrm{f} 25 \quad 12$ & f5 \\
\hline $18 \mathrm{f} 4$ & 10 & & & & & & & & & & \\
\hline s158 & 1.00 & TORS & 14 & 20 & 23 & 8 & $\mathrm{CNCN}$ & -179.14 & $\mathrm{f} 545 \quad 43$ & f545 35 & \\
\hline s159 & 1.00 & TORS & 47 & 53 & 56 & 41 & $\mathrm{CNCN}$ & -180.86 & $\mathrm{f} 545 \quad 35$ & $\mathrm{f} 545 \quad 40$ & \\
\hline k160 & 1.00 & TORS & 2 & 10 & 9 & 4 & NNCN & -0.46 & f687 49 & & \\
\hline s161 & 1.00 & TORS & 39 & 35 & 1 & 2 & CNFeN & 90.00 & f22 28 & & \\
\hline k162 & 1.00 & TORS & 35 & 43 & 42 & 37 & NNCN & 0.46 & f691 29 & & \\
\hline s163 & -1.00 & TORS & 6 & 2 & 10 & 9 & CNNC & 1.11 & $\mathrm{f} 52 \quad 19$ & f45 11 & \\
\hline
\end{tabular}




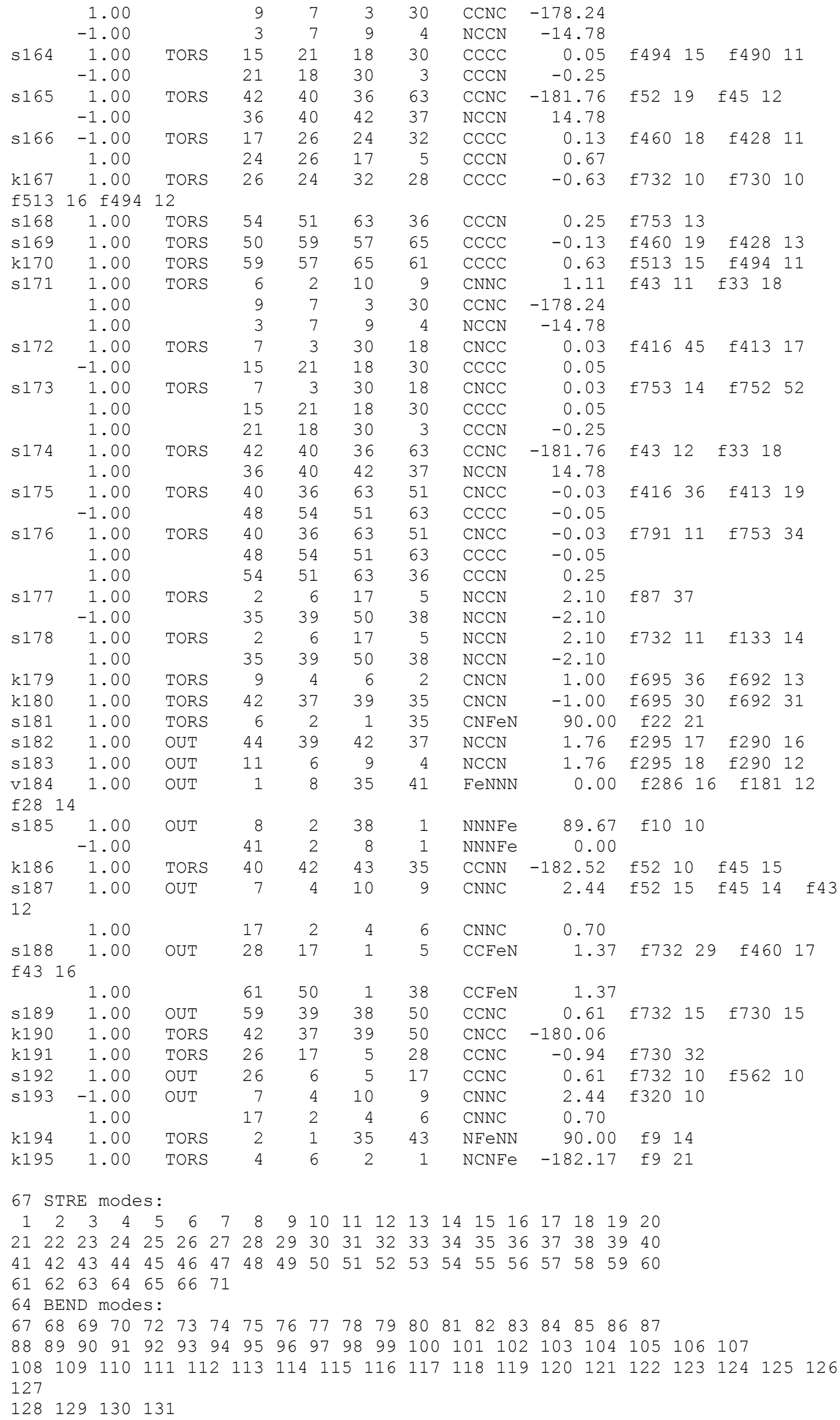




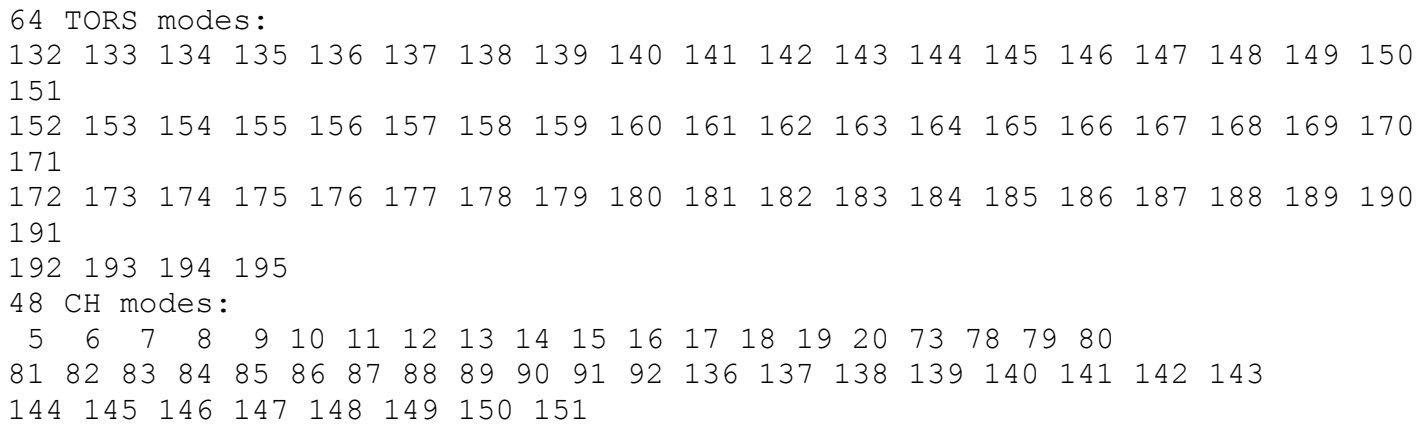

\section{Check of the Calculated Raman Spectra for the abpt Ligand and Complex 1}

Plots of the experimental peak frequencies vs the calculated ones for the case of the organic ligand and complex1 are shown in Figure S4.

The data appearing in the figures correspond to the frequencies of selected peaks which possess high intensity/high activity in the corresponding experimental/calculated spectra. For the experimental peaks the frequencies were obtained from the Raman spectrum acquired at the lowest temperature attained in our experiments (i.e. $83 \mathrm{~K}$ ). Linear relations were extracted between the correlation of calculated and experimental values. For both cases the slopes are similar.

abpt: $v_{\text {exp. }}=(0.977 \pm 0.002) v_{\text {calc. }}$

LS Fe(II) complex: $v_{\text {exp. }}=(0.979 \pm 0.001) v_{\text {calc. }}$
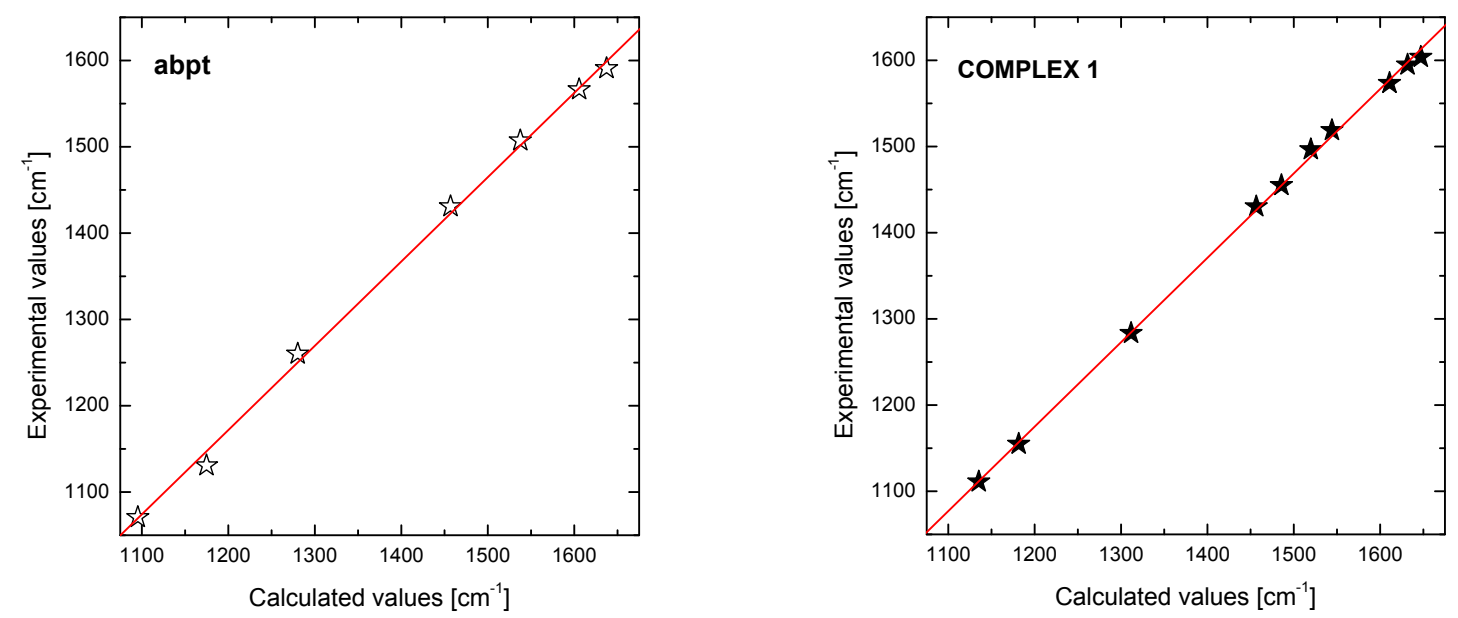

Figure S4. Linear relations between experimental and calculated Raman frequencies for (a) abpt and (b) complex1. 


\section{References}

S1.Jamroz, M.H., Vibrational Energy Distribution Analysis VEDA 4, Warsaw, 2004-2010. 